\title{
Searching for Periodic Variations in Nuclear Decay Rates using the NEMO-3 Detector
}

\author{
John Cesar (for the NEMO-3 and SuperNEMO Collaborations) ${ }^{* *}$ \\ The University of Texas at Austin \\ E-mail: jpcesar@utexas.edu
}

The NEMO-3 experiment searched for neutrinoless double-beta decay over the course of more than seven years utilizing various different candidate isotopes. Due to its multi-observable design it was able to isolate, with high fidelity, a very pure set of double-beta decay events with a signalto-background ratio of 76. Capitalizing on these two advantages, a search for time-dependent periodic variations in NEMO-3 nuclear decay rates is presented.

38th International Conference on High Energy Physics

3-10 August 2016

Chicago, USA

* Speaker.

${ }^{\dagger}$ A footnote may follow. 


\section{Motivation}

As an observatory which operated for over seven years with the ability to distinguish, with high fidelity, various nuclear decay processes, the NEMO-3 experiment has the potential to search for new physics phenomena by searching for time variations of fundamental constants or processes. In particular, variations in nuclear decay rates could point to changes in the fine structure constant $(\alpha)$ or the weak coupling constant. Such variations have already been postulated by [1] in which an annual modulation of beta decay rates is claimed. The authors also argue for potential correlations with modulations seen in other experiments such as the DAMA dark matter search experiment [2]. Searching for periodicities in the double-beta decay rate of ${ }^{100}$ Mo using the NEMO-3 detector would be the first such analysis to look for modulations in a second-order weak process. Furthermore, NEMO-3's ability to isolate different nuclear decays via its topological selection process can be used for further searches in other decay channels such as single-beta decay rates.

\section{The NEMO-3 Data Set}

The NEMO-3 detector and its approach in measuring two-neutrino double beta decay and searching for neutrinoless double beta decay is discussed in greater detail in the contribution to these proceedings from the NEMO-3 parallel presentation [3]. The baseline approach to this analysis involves using the established event selection procedure from [4] to produce a set of doublebeta decay events with a very high purity (signal to background ratio of 76). These events are then broken down by run so that the decay rate for each can be computed by taking the number of double-beta decays in a given run and dividing by the duration of that run.

The decay rates calculated in this way don't yet account for the detector efficiency. A full Monte-Carlo (MC) simulation of the detector response, as well as all run conditions which may effect event selection, is used to compare the number of events which are finally selected against the number of events generated for each run. The ratio of these is taken as the detector efficiency which is used to scale the measured run rates to calculate the true decay rate values.

\subsection{Blinding}

Before the scaled run decay rates are used to construct a time series, with the rates assigned to the midpoint times of corresponding runs, the analysis is kept blinded by performing various studies on a scrambled time series which uses true data but with the temporal ordering shuffled. The blinded time series corresponding to the double-beta decay rate of ${ }^{100}$ Mo for the second phase of detector operation is shown in figure 1 and spans 6.02 years.

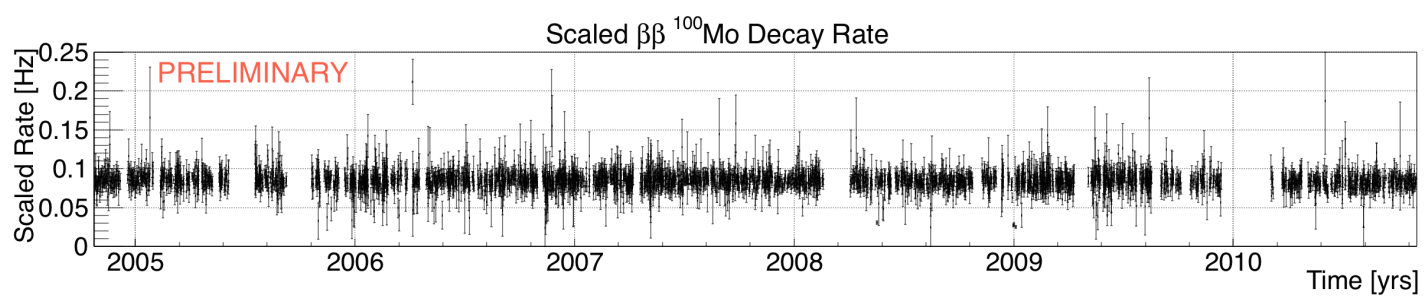

Figure 1: Temporally scrambled time series of the ${ }^{100}$ Mo double-beta decay rate spanning 6.02 years. 


\section{The Lomb-Scargle Periodogram Technique}

The periodogram technique is a common and robust method for searching for periodicities within a time series. After a Fourier transform into the frequency domain, a quantity known as the power is calculated for a sample frequency of interest. The periodogram is constructed by calculating this quantity over a range of sample frequencies and a large power at any particular frequency denotes a high likelihood for a modulation of the corresponding period hidden in the data.

The technique was extended by J. D. Scargle in [5] to work on unevenly sampled time series. M. Zechmeister and M. Kürster then further generalized the technique to account for data weights (via a $\chi^{2}$ approach) and potential offsets [6]. For an arbitrary time series $X\left(t_{i}\right)$ with $i=1,2,3, \ldots, N$ and uncertainties $\sigma\left(t_{i}\right)$ the calculation of the Generalized Lomb-Scargle (GLS) powers is given by

$$
\begin{aligned}
P_{G L S}(\omega)=\frac{1}{\sum_{i=1}^{N} w_{i}\left(X\left(t_{i}\right)-\bar{X}\right)^{2}}\left\{\frac{\left[\sum_{i=1}^{N} w_{i}\left(X\left(t_{i}\right)-\bar{X}\right) \cos \omega\left(t_{i}-\tau\right)\right]^{2}}{\sum_{i=1}^{N} w_{i} \cos ^{2} \omega\left(t_{i}-\tau\right)-\left[\sum_{i=1}^{N} w_{i} \cos \omega\left(t_{i}-\tau\right)\right]^{2}}\right. \\
\left.+\frac{\left[\sum_{i=1}^{N} w_{i}\left(X\left(t_{i}\right)-\bar{X}\right) \sin \omega\left(t_{i}-\tau\right)\right]^{2}}{\sum_{i=1}^{N} w_{i} \sin ^{2} \omega\left(t_{i}-\tau\right)-\left[\sum_{i=1}^{N} w_{i} \sin \omega\left(t_{i}-\tau\right)\right]^{2}}\right\}
\end{aligned}
$$

where $\omega$ is the sample frequency at which the power is being calculated, $\bar{X}$ is the weighted average of the $X\left(t_{i}\right)$ data points, $w_{i}=\frac{1}{\sigma\left(t_{i}\right)^{2}}$ are the weights, and the factor $\tau$ is given via the relation

$$
\tan (2 \omega \tau)=\frac{\sum_{i=1}^{N} w_{i} \sin \left(2 \omega t_{i}\right)-2 \sum_{i=1}^{N} w_{i} \sin \left(\omega t_{i}\right) \sum_{i=1}^{N} w_{i} \cos \left(\omega t_{i}\right)}{\sum_{i=1}^{N} w_{i} \cos \left(2 \omega t_{i}\right)-\left[\left(\sum_{i=1}^{N} w_{i} \sin \left(\omega t_{i}\right)\right)^{2}-\left(\sum_{i=1}^{N} w_{i} \cos \left(\omega t_{i}\right)\right)^{2}\right]}
$$

The expression for the $P_{G L S}(\omega)$ can be normalized such that the distribution of powers follows an exponential relation with unit mean. Then the probability of finding a power $Z$ larger than $\mathrm{z}$ is given by $P(Z>z)=\exp (-z)$ so one can estimate the significance of any peak via its confidence level (C.L.) by the relation C.L. $=100 \times[1-\exp (-z)]^{M}$ where $\mathrm{M}$ is the number of sample frequencies.

\subsection{GLS Example}

An example of the Generalized Lomb-Scargle (GLS) periodogram technique is shown in figure 2. An arbitrary time series of pure Gaussian noise terms $X\left(t_{i}\right)$ was generated upon which a sinusoidal modulation was applied in the form $X^{\prime}\left(t_{i}\right)=X\left(t_{i}\right) \times\left[1+A \cos \left(2 \pi f t_{i}\right)\right]$. The relative amplitude was set to $5 \%(A=0.05)$ while the period was set to $10(f=0.1)$, in arbitrary time units. This time series is shown in the top plot with the modulation function shown in red.

The GLS periodogram, shown in the bottom plot of figure 2, was calculated for 1000 evenly spaced sample frequencies between $[0,1]$. A large peak can be seen with GLS power over 20 at $\omega=0.09987$ corresponding to a period of 10.02. This matches the input modulation frequency with an error of only $0.2 \%$. Furthermore, a high C.L. value $(99.97 \%)$ is attributed to this peak.

\section{Sensitivity Studies}

Studies were also performed to determine the expected sensitivity to detecting a periodic signal. This was done by generating many MC data sets which mimic true NEMO-3 data for doublebeta decays originating in the ${ }^{100} \mathrm{Mo}$ foils. For each MC time series, a modulation was applied 


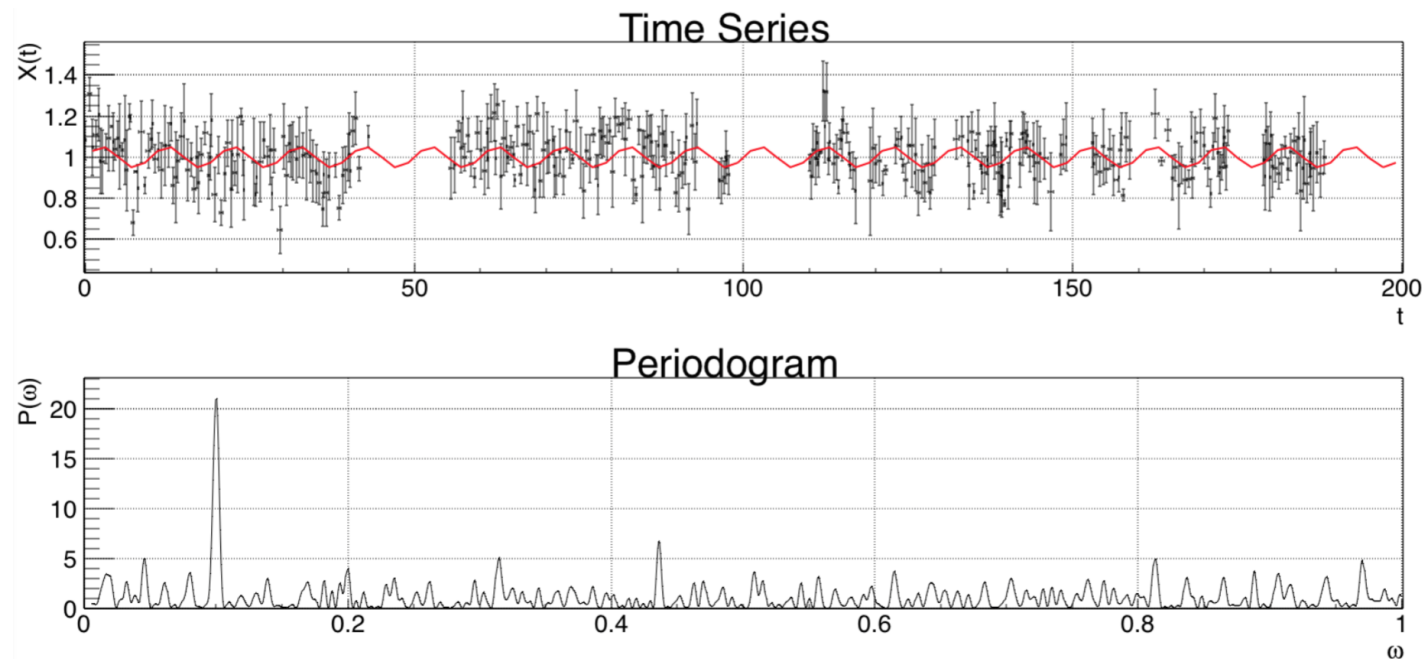

Figure 2: An example application of the GLS technique using an arbitrary modulated time series (top plot). The resultant periodogram, with a large peak at the input modulation frequency, is shown in the bottom plot.

(similar to in the example above) with various values of A and $\mathrm{f}$ (broken down into three regimes). The GLS technique was applied to each modulated data set and the largest periodogram peak was recorded along with its significance (C.L. value). The sensitivity plots in figure 3 show how the detection sensitivity (in terms of peak significance) changes as $\mathrm{A}$ and $\mathrm{f}$ are varied.
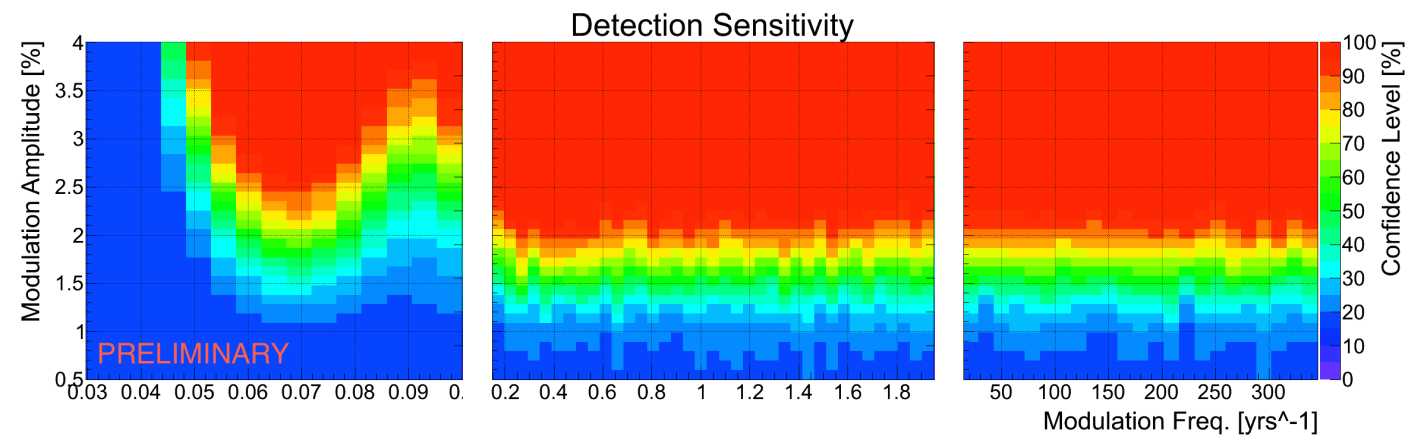

Figure 3: Detection sensitivity as a function of modulation amplitude and frequency for double-beta decays in ${ }^{100}$ Mo foils.

\section{References}

[1] J. H. Jenkins, et al., Astropart. Phys. 32 (2009) 42-46.

[2] R. Bernabei, et al., Eur. Phys. J. C 73 (2013) 2648.

[3] J. P. Cesar, et al., these proceedings.

[4] R. Arnold, et al., Phys. Rev. D 89 (2014) 111101.

[5] J. D. Scargle, Astrophys. J. 263 (1982) 835-853.

[6] M. Zechmeister, M. Kürster, Astro. \& Astrophys. 496 (2009) 577-584. 